\title{
Clopidogrel hypersensitivity: clinical challenges and options for management.
}

\author{
Kimberly L Campbell \\ Thomas Jefferson University \\ John R Cohn \\ Thomas Jefferson University \\ Michael P Savage \\ Thomas Jefferson University
}

Follow this and additional works at: https://jdc.jefferson.edu/cardiologyfp
Let us know how access to this document benefits you

\section{Recommended Citation}

Campbell, Kimberly L; Cohn, John R; and Savage, Michael P, "Clopidogrel hypersensitivity: clinical challenges and options for management." (2010). Division of Cardiology Faculty Papers. Paper

13.

https://jdc.jefferson.edu/cardiologyfp/13

This Article is brought to you for free and open access by the Jefferson Digital Commons. The Jefferson Digital Commons is a service of Thomas Jefferson University's Center for Teaching and Learning (CTL). The Commons is a showcase for Jefferson books and journals, peer-reviewed scholarly publications, unique historical collections from the University archives, and teaching tools. The Jefferson Digital Commons allows researchers and interested readers anywhere in the world to learn about and keep up to date with Jefferson scholarship. This article has been accepted for inclusion in Division of Cardiology Faculty Papers by an authorized administrator of the Jefferson Digital Commons. For more information, please contact: JeffersonDigitalCommons@jefferson.edu. 


\title{
As submitted to:
}

\section{Expert Review of Clinical Pharmacology}

And later published as:

\section{Clopidogrel Hypersensitivity: Clinical Challenges and Options for Management}

\author{
Volume 3, Issue 4, July 2010, Pages 553-561 \\ DOI: 10.1586/ecp.10.30
}

\begin{abstract}
Kimberly L. Campbell, M.D., John R. Cohn, M.D., and Michael P. Savage, M.D. Thomas Jefferson University Hospital, Division of Cardiology 925 Chestnut Street, Mezzanine level; Philadelphia, PA 19107

Tel: (215) 955-6478, Fax: (215) 923-4942

Michael.Savage@jefferson.edu
\end{abstract}

\begin{abstract}
:
Over 90 million patients have been prescribed clopidogrel since its FDA approval in 1997. Clopidogrel hypersensitivity affects $6 \%$ of patients, most commonly in the form of a pruritic rash. Symptoms are severe enough to result in drug discontinuation in $1.5 \%$. Premature discontinuation of clopidogrel is problematic after percutaneous coronary intervention because of the risk of stent thrombosis and death. Accordingly, the management of patients with clopidogrel hypersensitivity is of significant clinical importance. Conventional clopidogrel desensitization protocols have been successful, but require a washout period off medication to enable accurate detection of reaction to during desensitization. This technique is therefore undesirable in patients following stent placement. Clopidogrel hypersensitivity can be successfully treated without drug interruption using short-course corticosteroids and antihistamines to enable development
\end{abstract}


of physiologic tolerance while the medication is continued. The role of newer agents, such as prasugrel, as surrogate therapy in patients with clopidogrel hypersensitivity is yet to be defined.

Short title: Clopidogrel Hypersensitivity

Keywords: clopidogrel, hypersensitivity, drug allergy, anti-platelet therapy, desensitization, thienopyridine.

Clopidogrel is the most frequently prescribed antiplatelet therapy, with more than 9.1 million prescriptions filled in 2008. Clopidogrel, a member of the thienopyridine class of antiplatelet agents, directly inhibits adenosine diphosphate (ADP)-induced platelet aggregation by irreversibly binding the $\mathrm{P} 2 \mathrm{Y}_{12}$ receptor. The oral prodrug is converted into the active metabolite by the cytochrome P-450 pathway in the liver. Clopidogrel has become widely prescribed for prevention of vascular events following myocardial infarction (MI), cerebrovascular accident (CVA), and in patients with known peripheral arterial disease (PAD).

Several large studies have demonstrated the benefits of clopidogrel therapy following MI and stroke. The Clopidogrel versus Aspirin in Patients at Risk of Ischemic Events (CAPRIE) trial randomized 19,185 patients with recent MI, CVA, or documented PAD to receive either clopidogrel $75 \mathrm{mg}$ daily or aspirin $325 \mathrm{mg}$ daily [1]. Clopidogrel was associated with an $8.7 \%$ relative risk reduction in the composite endpoint of stroke, MI, or vascular death. The Clopidogrel in Unstable Angina to Prevent Recurrent Events (CURE) trial demonstrated further benefit from clopidogrel in combination with aspirin [2]. In this study, 12,562 patients with non-ST elevation acute coronary syndrome were 
randomized to either clopidogrel $75 \mathrm{mg}$ daily or placebo in addition to aspirin therapy. Compared to aspirin monotherapy, dual antiplatelet therapy resulted in $20 \%$ relative risk reduction in the composite endpoint of cardiovascular death, nonfatal MI, and nonfatal CVA.

Clopidogrel is also widely prescribed in combination with aspirin following percutaneous coronary intervention (PCI) for the prevention of stent thrombosis. Premature discontinuation of dual antiplatelet therapy following PCI is the most important clinical predictor of acute stent thrombosis. Early thienopyridine discontinuation is associated with a 30-fold rise in subacute stent thrombosis, which typically results in $\mathrm{MI}$ and is associated with a 20 to $45 \%$ mortality $[3,4,5,6]$. The Clopidogrel Aspirin Stent International Cooperatives Study (CLASSICS) and Ticlid or Plavix Post Stent (TOPPS) trials showed clopidogrel was non-inferior to ticlopidine in reducing major adverse cardiovascular events (MACE) and acute stent thrombosis $[7,8]$. Because of its improved safety profile, clopidogrel quickly replaced ticlopidine as the thienopyridine of choice following PCI. With the advent of drug-eluting stents, and the Clopidogrel in Unstable Angina to Prevent Recurrent Ischemic Events (CURE) trial, the recommended duration of clopidogrel therapy following PCI has increased from the initial 4 weeks to a minimum of 12 months [9].

\section{Clopidogrel hypersensitivity}

Allergic reaction to clopidogrel most frequently presents as pruritic rash and occurs in $4-6 \%$ of patients receiving clopidogrel $[1,10]$. Rash is severe enough to result in drug discontinuation in $1.5 \%$ of patients [1]. More severe hypersensitivity syndromes 
have been reported including febrile illness [11,12], leucopenia [13,14], thrombotic thrombocytopenic purpura $[15,16,17,18]$, aplastic anemia $[19,20,21,22]$, angioedema [23], serum sickness [24], systemic inflammatory response syndrome [25], and end organ injury [26]. These more serious hypersensitivity reactions are rare, and morbiliform rash remains the most frequently encountered reaction to clopidogrel therapy.

In the largest published case series of 24 consecutive patients presenting with clopidogrel hypersensitivity, von Tiehl et al observed that the median onset of hypersensitivity symptoms was 6 days after initiation of therapy [27]. The rash was macular and erythematous, beginning on the face, chest or abdomen. With progression, it became a confluent generalized rash that involved the palms and soles in $29 \%$ of patients. Similar observations were made in our cohort of 20 patients experiencing clopidogrel hypersensitivity after undergoing PCI [28]. Mean onset of symptoms was $6 \pm 2$ days after clopidogrel exposure. The distribution of time to onset of hypersensitivity symptoms is shown in Figure 1. Ninety percent of these patients presented with the typical erythematous pruritic rash described by von Tiehl. Additional symptoms of clopidogrel hypersensitivity observed in this cohort included non-pruritic rash and angioedema [28].

Diagnosing clopidogrel hypersensitivity can be challenging. Antibody-based laboratory assays for clopidogrel hypersensitivity are not available. Clinically, the diagnosis can be made from symptom resolution with drug discontinuation and recurrence with repeat challenge. Clopidogrel hypersensitivity typically presents during the first two weeks of therapy, coinciding with the time of highest risk for stent thrombosis. In this setting of recent PCI, clopidogrel discontinuation carries a 25-29\% 
risk for stent thrombosis $[4,6]$. Therefore, clinical diagnosis must often be made based on history and temporal relationship to onset of symptoms. Definitive clinical diagnosis is further complicated by the concomitant introduction of multiple medications, iodinated contrast, and drug impregnated polymer stents.

The exact mechanism of clopidogrel hypersensitivity is not clearly understood.

True hypersensitivity reactions result from an IgE-mediated immunologic response to an antigen. Vigo et al report a case of clopidogrel hypersensitivity in a patient with a positive intradermal skin test response to clopidogrel [29]. Skin biopsies in clopidogrel hypersensitivity reveal nonspecific findings compatible with drug eruption [11]. This would suggest an IgE-mediated process. However, there may also be a role for direct mast-cell or complement activation, immune complex formation, or T-cell activation by clopidogrel or one of its metabolites.

\section{Pharmacologic alternatives to clopidogrel}

Dual anti-platelet therapy with aspirin and clopidogrel has become the standard of care after PCI with stent placement [30]. For patients with allergy to clopidogrel, therapeutic alternatives are limited. Until recently, ticlopidine was the only alternative thienopyridine class antiplatelet agent available. Ticlopidine, like clopidogrel, is proven efficacious in reducing bare metal stent thrombosis and preventing cardiovascular events after stroke $[31,32,33]$. There is limited data on the use of ticlopidine with drug-eluting stents [34]. The clinical utility of ticlopidine is limited by the occurrence of gastrointestinal side effects, neutropenia, thrombocytopenia, and rash. Up to $21 \%$ of patients on ticlopidine discontinue therapy due to these side effects $[31,33,35]$. 
Neutropenia occurs in $2.4 \%$ of patients [35], and thrombotic thrombocytopenic purpura (TTP) has been reported with an incidence of $0.02 \%$ [36]. As a result of these hematologic side effects, CBC monitoring every 2 weeks is necessary for patients receiving ticlopidine.

In July 2009 the FDA approved prasugrel, the newest thienopyridine and alternative to clopidogrel therapy. In TRITON-TIMI 38, prasugrel was associated with a $19 \%$ relative risk reduction in cardiovascular death, nonfatal MI, and nonfatal CVA compared to clopidogrel. This reduction in cardiovascular events was counter-balanced by an increase in the risk of life-threatening bleeding (1.4\% versus $0.9 \%)$ [37]. In patients with history of transient ischemic attack or stroke, the risk of intra-cranial hemorrhage overpowered the benefits of prasugrel. The use of prasugrel is contraindicated in these patients. Additional groups at increased risk for bleeding with prasugrel include patients greater than 75 years of age, less than $60 \mathrm{~kg}$ in weight, and those who have propensity to bleed or require use of other medications increasing the risk of bleeding [38].

Cross-reactivity between clopidogrel and ticlopidine has been described in several case reports $[27,39,40,41]$. The two medications differ only by the substitution of a carboxymethyl side group on clopidogrel [42]. The chemical structures of the clinically available thienopyridine antiplatelet agents are shown in Figure 2. It is unclear which moiety of the clopidogrel and ticlopidine stimulates the allergenic response. Whether this cross-reactivity carries over to prasugrel has yet to be determined and the use of this new agent in patients with clopidogrel hypersensitivity has not been reported. 
For patients in whom alternative thienopyridine class antiplatelet agents are contraindicated, there is no proven non-thienopyridine therapeutic alternative. The use of warfarin with aspirin for bare metal stents was evaluated in the Full Anticoagulation versus Aspirin and Ticlopidine (FANTASTIC) study [43]. This study randomized 485 patients to receive either ticlopidine $250 \mathrm{mg}$ twice daily or warfarin adjusted to an INR of 2.5-3.0 for 6 weeks after PCI. Warfarin therapy resulted in a 50\% increase in bleeding complications without affecting the rate of MI, death, or stent occlusion. In a second trial, 517 patients were randomized to warfarin or ticlopidine plus aspirin [44]. A higher incidence of cardiac events was noted in the warfarin group in addition to the previously seen increase in hemorrhagic events and vascular complications.

The largest trial, Stent Anticoagulation Restenosis Study (STARS), enrolled 1653 patients and compared aspirin, aspirin with ticlopidine, and aspirin with warfarin [31]. Aspirin monotherapy and in combination with warfarin were both inferior to aspirin with ticlopidine in reducing major adverse cardiac events and stent thrombosis. The risk of hemorrhagic complications was highest in those patients receiving anticoagulation with warfarin. Although warfarin with aspirin was used before thienopyridines were available, these studies have established that warfarin is inferior to dual antiplatelet therapy in the reduction of cardiovascular events following PCI.

Several randomized trials have investigated cilostazol as an alternative to thienopyridines in patients receiving bare metal stents. The Randomized Prospective Antiplatelet Trial of Cilostazol versus Ticlopidine in Patients Undergoing Coronary Stenting (RACTS) evaluated ticlopidine 250mg twice daily or cilostazol 100mg twice daily in 397 patients [45]. At 9 months, there was no significant difference in death, MI, 
stroke, and stent thrombosis. A second trial by Lee et al found similar results comparing cilostazol against clopidogrel [46]. They observed no significant difference in targetlesion revascularization or drug-related adverse events. In a third trial comparing cilostazol versus ticlopidine, there was no significant difference at 6 months in restenosis or coronary angiographic result [47]. However, at 3 years, the group treated with cilostazol experienced significantly less death, MI, stent thrombosis, revascularization, and CVA. There is limited data on the use of cilostazol following drug eluting stent placement. The use of cilostazol is contraindicated in patients with heart failure, and caution must be used in patients who take inhibitors of the cytochrome P450 system.

\section{Clopidogrel desensitization}

The mechanism of drug desensitization is complex and has yet to be fully elucidated. One theory relates the success of desensitization to alterations in the balance of circulating $\operatorname{IgE}$ and $\operatorname{IgG}$ levels [48]. Following antigen exposure, there is a transient rise in $\operatorname{IgE}$ antibodies, but also a rise in levels of $\mathrm{IgG}$ antibodies. These allergen-specific IgG antibodies activate a negative feedback loop inhibiting IgE-dependent histamine release by basophils and $\mathrm{T}$ cell activation. Desensitization also appears to shift the balance of T helper cells towards a Th1 population and away from the Th2 subtype that drives allergen responses [48]. A second theory proposes a state of mast cell unresponsiveness develops from gradual antigen exposure. By supplying antigen in small incremental doses, the IgE receptors are slowly bound and occupied without triggering mast cell activation [40]. Likely, both of these pathways contribute to the success of desensitization. 
Traditional desensitization protocols, similar to those used for aspirin and antibiotics, have been used with success in patients with clopidogrel hypersensitivity (Table 1). These protocols rely on withdrawal of clopidogrel to allow hypersensitivity symptoms to resolve prior to initiation of desensitization. Beta blockers, antihistamines, and steroids are held for 48-96 hours before desensitization. Patients are administered escalating doses of clopidogrel starting at 0.005 to $0.02 \mathrm{mg}$ and doubling every 15 to 30 minutes until the target dose of $75 \mathrm{mg}$ is achieved. If hypersensitivity symptoms recur during desensitization, symptoms are treated and protocol is held until symptoms resolve. The protocol is resumed by repeating the inciting dose. Desensitization is typically completed in 3-8 hours.

The first case report of successful clopidogrel desensitization was presented at the American College of Allergy, Asthma, and Immunology Annual Meeting in 2004. Vigo et al describe a patient who experienced typical clopidogrel hypersensitivity rash on the $5^{\text {th }}$ day of clopidogrel therapy [29]. Skin testing confirmed a reaction to clopidogrel and the patient was successfully desensitized with escalating doses of clopidogrel starting at $0.02 \mathrm{mg}$ and administered every 15 minutes.

During the same time, Camara and Almeda were successfully desensitizing patients using an 8-hour protocol starting at $0.005 \mathrm{mg}$ and administering escalating doses of clopidogrel every 30 minutes. They report on successful inpatient and outpatient desensitization in 3 patients presenting with clopidogrel hypersensitity rash [40]. Two of the three patients in this case-series had experienced hypersensitivity with both clopidogrel and ticlopidine, necessitating desensitization. Following this same protocol, Lee-Wong was able to reproduce their success with clopidogrel desensitization on an 
additional patient [49]. All subsequent desensitization protocols have been modifications derived from the protocols described by Vigo or Camara and Almeda.

Walker et al described their experiences in 8 patients with an accelerated 3 hour desensitization protocol at their institution [39]. Based on the findings of Vigo, they started desensitization with a dose of $0.02 \mathrm{mg}$ and administered escalating 2-fold dosages every 15 minutes in a monitored ICU setting. In contrast to previous desensitization protocols that restricted treatment to patients with cutaneous manifestations of hypersensitivity, they included 2 patients experiencing more the more severe hypersensitivity reaction of angioedema. All 8 patients were successfully desensitized without major adverse events. A follow-up report described further success in desensitization of an additional 5 patients [50].

The largest published case series involved 24 consecutive patients with clopidogrel hypersensitivity following drug-eluting stent implantation [27]. Patients were desensitized by von Tiehl et al using an 8-hour protocol modified from Camara and Almeda [40]. In contrast to other case reports, patients were bridged through the washout period using ticlopidine, a thienopyridine similar in structure to clopidogrel. At least one of the patients experienced similar hypersensitivity reaction to ticlopidine.

Desensitization was performed starting at a dose of $0.005 \mathrm{mg}$ with incremental doubling doses administered every 30 minutes. Initial desensitization was successful in all patients, with 4 of the 24 patients requiring treatment for cutaneous reactions during desensitization. Two patients (8\%) required repeat desensitization - one for persistent rash, and one for recurrent rash following 3 days of noncompliance. Since von Tiehl's 
published case series, additional case reports continue to demonstrate the success of this desensitization protocol $[51,52]$.

Traditional escalating dose desensitization protocols have been shown effective in treating clopidogrel hypersensitivity manifest as cutaneous rash and angioedema. There have been no reports using this technique in patients whom experience gastrointestinal or hematologic side effects, anaphylaxis, or systemic hypersensitivity syndromes. In the appropriately selected patient, this desensitization protocol can be safely performed in the outpatient setting $[27,50]$.

\section{Management of hypersensitivity reaction without clopidogrel interruption}

In a retrospective analysis at our institution, 20 patients were identified that experienced clopidogrel hypersensitivity following bare metal or drug eluting stent implantation [28]. The most common symptom was rash, but one patient presenting with angioedema was included. In a novel approach, patients were treated for their hypersensitivity symptoms without interrupting clopidogrel therapy. Treatment regimen included antihistamines alone in 5 patients, corticosteroids alone in 4 patients, and combination of antihistamines and corticosteroids in 11 patients. The most commonly

prescribed corticosteroid regimen was a 6 day solumedrol taper (Medrol Dosepak), with the mean corticosteroid therapy duration being $9 \pm 6$ days (range 6-20).

Treatment of clopidogrel hypersensitivity with antihistamines and short-course corticosteroids was successful in 17 patients (85\%). Most patients were successfully treated as outpatients $(80 \%)$. Two patients experienced recurrence of their symptoms, which were successfully treated with a longer corticosteroid taper and the addition of a 
leukotriene inhibitor. The three patients in whom this approach was unsuccessful included one patient with angioedema, one patient with progression to desquamation, and one patient with intolerable pruritis.

The 17 patients who were successfully treated were able to complete the minimum recommended duration of clopidogrel therapy without drug interruption. The mean duration of clopidogrel therapy in these patients is $388 \pm 272$ days, with 843 days being the longest duration of clopidogrel therapy. As of last follow-up, $60 \%$ of these patients remained on long-term clopidogrel therapy. There was no stent thrombosis, death, myocardial infarction, or stroke during treatment or follow-up.

In a similar strategy, Cheema et al treated 25 patients presenting with clopidogrel hypersensitivity rash with oral corticosteroids while continuing clopidogrel therapy [53]. Patients completed a 2 week tapering course of prednisone starting at $30 \mathrm{mg}$ twice daily. All 25 patients were successfully treated, without recurrence of symptoms. By suppressing the symptoms of clopidogrel hypersensitivity with antihistamines and corticosteroids, patients are able to develop physiologic tolerance without interrupting clopidogrel therapy. These two series suggest short-course corticosteroids and antihistamines offer a safe and effective alternative to traditional desensitization protocols. This method appears to allow the development of physiologic tolerance without interrupting drug therapy. Our current approach at the Thomas Jefferson University Hospital for management of clopidogrel hypersensitivity is summarized in Table 2.

\section{Expert commentary}


Diagnosing hypersensitivity is complex, with new medications and drug impregnated polymer stents frequently introduced during the same admission following a cardiovascular event. Both immunological and non-immunological mechanisms contribute to adverse drug events, and the exact pathophysiology of hypersensitivity remains unknown. In most cases there are no reliable diagnostic tests beyond drug challenge and history. In hypersensitivity reactions that develop shortly after stent implantation, it is often difficult to distinguish between the stent, aspirin, or clopidogrel as the source of the reaction. One must heavily rely on clinical history for the identification of the inciting agent in this population.

The management of clopidogrel hypersensitivity is challenging, particularly in the post PCI population. In patients receiving clopidogrel for secondary cardiovascular prevention, traditional desensitization protocols are highly successful to enable resumption of clopidogrel therapy. In contrast, PCI patients typically experience hypersensitivity during the first two weeks of clopidogrel therapy when drug discontinuation carries the greatest risk for stent thrombosis. In this population, a traditional desensitization protocol incorporating a pharmacologic wash out period is a less viable option. Treatment of clopidogrel hypersensitivity with tapering doses of corticosteroids and antihistamines is successful in $85-100 \%$ of cases, and appears to be as successful as traditional desensitization protocols.

With the FDA approval of prasugrel, there is now a more viable alternate thienopyridine class antiplatelet agent. Use of ticlopidine, the first available thienopyridine, was clinically limited by frequent side effects and adverse reactions. Although still available by prescription, ticlopidine is often not tolerated long enough to 
complete adequate therapy following drug-eluting stent placement. Prasugrel is more efficacious than clopidogrel in reducing cardiovascular death, MI, and stroke but incurs a higher risk of bleeding. For this reason, it is not a therapeutic option in patients with prior cerebrovascular events. Cross-reactivity among thienopyridines further limits the use of therapeutic alternatives in clopidogrel hypersensitivity. Use of cilostazol or warfarin as sufficient alternatives to thienopyridine therapy has not been established.

In the post PCI population, treatment of clopidogrel hypersensitivity with corticosteroids and antihistamines remains a viable option, especially in patients unable or ineligible to take the alternative thienopyridine prasugrel. The elimination of the washout period avoids the risk of catastrophic stent thrombosis due to early discontinuation of antiplatelet therapy. Successful treatment enables long-term continuation of therapy and confers a low risk of adverse cardiovascular events. Lifethreatening or severe hypersensitivity syndromes including Stevens - Johnson syndrome and anaphylaxis should prompt consideration of the risks and benefits of desensitization versus alternative therapies.

\section{Five-year view}

Newly available in the U.S., prasugrel is a viable alternative to clopidogrel in patients without a history of cerebrovascular event, low body mass, or advanced age. As Sanofi Aventis's patent for clopidogrel bisulfate expires in November 2011, generic equivalents of clopidogrel will become available in the United States. With the advantage of reduced cost and lower incidence of bleeding, it will likely remain first line therapy in the prevention of stent thrombosis and prevention of secondary cardiovascular 
events over alternative thienopyridines. Furthermore, the use of clopidogrel may be extended in the future should prolonged thienopyridine therapy (beyond one year) prove to be beneficial in patients with drug-eluting stents.

It is reasonable to expect some cross-hypersensitivity between clopidogrel and prasugrel, as has been documented to occur with ticlopidine. As prescriptions for clopidogrel rise, more physicians including family practitioners, internist, neurologists, and cardiologists will be faced with treating clopidogrel hypersensitivity. As clinicians, we will continue to face the challenges of clopidogrel hypersensitivity and the complexities of its management. Further studies will be necessary to determine if prasugrel is a safe and effective substitute for clopidogrel in patients with hypersensitivity reactions.

The mechanisms of drug hypersensitivity are complex and not fully understood. IgE mediated mast cell response is believed to play a role in the process. Escalating dose desensitization protocols are an effective means of overcoming hypersensitivity reactions. The mechanism of desensitization to achieve tolerance remains speculation. The success of the approach of enabling the development of physiology tolerance while continuing drug therapy raises the possibility of alternative pathways to drug desensitization. As this technique is proven successful in the management of clopidogrel hypersensitivity, it may be applicable in the future to other drug hypersensitivities.

\section{Key Issues}


- Clopidogrel, a thienopyridine class antiplatelet agent, remains a mainstay in the medical therapy for prevention of stent thrombosis and secondary cardiovascular events.

- Hypersensitivity to clopidogrel often manifests as a macularpapular rash starting on the trunk and spreading to the extremities, starting approximately a week after initiation of clopidogrel.

- Other thienopyridines include ticlopidine and recently prasugrel. Ticlopidine is poorly tolerated due to GI side effects and neutropenia, and prasugrel is associated with increased risk of bleeding.

- Non-thienopyridine alternatives including aspirin monotherapy and warfarin appear inferior to regimens including thienopyridines in the post PCI population.

- Traditional desensitization protocols utilizing a washout period are successful, but potentially expose patients to an increased risk of stent thrombosis.

- Future research will elucidate the mechanisms of drug hypersensitivity and the mechanism by which desensitization is effective.

- A novel approach to treating clopidogrel hypersensitivity with antihistamines and short-course corticosteroids is successful at enabling patients to develop physiologic tolerance without interruption in drug therapy.

- Application of this approach to other drug hypersensitivities will determine if this method of management is unique to clopidogrel hypersensitivity or can be applied to the management of other hypersensitivity reactions.

\section{Financial Disclosure / Acknowlegements}




\section{The authors have no financial disclosures to make.}

${ }^{1}$ CAPRIE Steering Committee. A randomised, blinded, trial of clopidogrel versus aspirin in patients at risk of ischemic events (CAPRIE). Lancet. 348,1329-1339 (1996).

* This international trial enrolled 19,185 patients randomized to receive either aspirin 325mg or clopidogrel $75 \mathrm{mg}$ once daily. This study demonstrated clopidogrel's superiority over aspirin in reducing the endpoint of combined end point of stroke, MI, or vascular death. It is the largest study evaluating the overall safetyprofile of clopidogrel.

${ }^{2}$ CURE Trial Investigators. Effects of clopidogrel in addition to aspirin in patients with acute coronary syndrome without st-segment elevation. N Engl J Med. 345,494-502 (2001).

${ }^{3}$ Cutlip DE, Baim DS, Ho KKL, et al. Stent thrombosis in the modern era: a pooled analysis of multicenter coronary stent clinical trials. Circulation. 103,1967-1971 (2001).

* This is a meta-analysis of six trials and non-randomized registries enrolling a total of 6,186 patients receiving one or more coronary stents, designed to evaluate the incidence of and factors relating to the development of stent thrombosis in the era of dual antiplatelet therapy.

${ }^{4}$ Jeremias A, Sylvia B, Bridges J, et al. Stent thrombosis after successful sirolimus-eluting stent implantation. Circulation. 109,1930-1932 (2004).

${ }^{5}$ Spertus, JA, Kettelkamp R, Vance C, et al. Prevalence, predictors, and outcomes of premature discontinuation of thienopyridine therapy after drug-eluting stent placement: results form the PREMIER Registry. Circulation. 113,2803-2809 (2006).

${ }^{6}$ Iakovou I, Schmidt T, Bonizzoni E, et al. Incidence, predictors, and outcome of thrombosis after successful implantation of drug-eluting stents. JAMA.. 293,2126-2130 (2005).

${ }^{7}$ Bertrand ME, Rupprecht H-J, Urban P, Gershlick AJ. Double-blind study of the safety of clopidogrel with and without a loading dose in combination with aspirin compared with ticlopidine in combination with aspirin after coronary stenting. The Clopidogrel Aspirin Stent International Cooperative Study (CLASSICS). Circulation. 102,624-629 (2000).

${ }^{8}$ Taniuchi M, Kurz HI, Lasala JM. Randomized comparison of ticlopidine and clopidogrel after intracoronary stent implantation in a broad patient population. Circulation 2001;104:539-543. 
${ }^{9}$ Steinhubl SR, Berger PB, Mann JT, et al for the CREDO Investigators. Early and sustained dual oral antiplatelet therapy following percutaneous coronary intervention: a randomized controlled trial. JAMA. $288,2411-2420$ (2002).

${ }^{10}$ Plavix (clopidogrel bisulfate) package insert. New York, NY: Bristol Myers Squibb Company \& SanofiSynthelabo, revised October 2007.

${ }^{11}$ Comert A, Akgun S, Civelek A, Kavala M, Sarigul S, Yilirim T, Arsan S. Clopidogrel-induced hypersensitivity syndrome associated with febrile pancytopenia. Int J Dermatol. 44,882-884 (2005).

${ }^{12}$ Sarrot-Reynauld F, Bouillet L, Bourrain J-L. Severe hypersensitivity associated with clopidogrel. Ann Intern Med. 135,305-306 (2001).

${ }^{13}$ Akcay A, Kanbay M, Agca E, Sezer S, Ozdemir FN. Neutropenia due to clopidogrel in a patient with end-stage renal disease [letter]. Ann Pharmacother. 38,1538-1539 (2004).

${ }^{14}$ McCarthy MW, Kocker DR. Clopidogrel-associated leucopenia. Ann Pharmacother 2003;37:216-219.

${ }^{15}$ Zakarija A, Bandarenko N, Pandey DK, et al. Clopidogrel-associated TTP: An Update of pharmacovigilance efforts conducted by independent researchers, pharmaceutical suppliers, and the food and drug administration. Stroke. 35,533-537 (2004).

${ }^{16}$ Connors JM, Gopfert C. Robson S, Churchill WH, Benjamin RJ. Clopidogrel associated TTP [abstract]. Transfusion. 39,s565 (1999).

${ }^{17}$ Bennett CL, Connors JM, Carwile JM, et al. Thrombotic thrombocytopenic purpura associated with clopidogrel. N Engl J Med. 342,1773-1777 (2000).

${ }^{18}$ Carwile JM, Laber DA, Soltero ER, et al. Thormbotic thrombocypenic purpura occurring after exposure to clopidogrel [abstract]. Blood. 94s1,78b (1999).

${ }^{19}$ Doogue MP, Begg EJ, Bridgman P. Clopidogrel hypersensitivity syndrome with rash, fever, and neutropenia. Mayo Clin Proc. 80,1368-1370 (2005).

${ }^{20}$ Meyer B. Staudinger T, Lechner K. Clopidogrel and aplastic anaemia [letter]. Lancet. 357,1446-1447 (2001).

${ }^{21}$ Trivier JM, Caron J, Mahieu M, Cambier N, Rose C. Fatal aplastic anemia associated with clopidogrel [letter]. Lancet. 357,446 (2001). 
${ }^{22}$ Andres E, Perrin AE, Alt M, Goichot B, Schlienger JL. Febrile pancyptopenia associated with clopidogrel. Arch Intern Med. 161,125 (2001).

${ }^{23}$ Fischer RC, Worm M, Groneberg DA. Clopidogrel-associated angioedema. Am J Medicine. 114,77-78 (2003).

${ }^{24}$ Phillips EJ, Knowles SR, Shear NH. Serum sickness-like reaction associated with clopidogrel [letter]. Br J Clin Pharmacol. 56,583 (2003).

${ }^{25}$ Wolf I, Mouallem M, Rath S, Farfel Z. Clopidogrel-induced systemic inflammatory response syndrome. Mayo Clin Proc. 78,618-620 (2003).

${ }^{26}$ Tholl U, Anlauf M, Helmchen U. Clopidogrel and membranous nephropathy [letter]. Lancet. 354,14431444 (1999).

${ }^{27}$ von Tiehl KF, Price MJ, Valencia R, Ludington KJ, Teirstein PS, Simon RA. Clopidogrel desensitization after drug-eluting stent placement. J Am Coll CardioI. 50,2039-2043 (2007).

** This is the largest published case series of 25 patients with clopidogrel hypersensitivity and describes the success of a traditional desensitization protocol.

${ }^{28}$ Campbell KL, Mallya R, Jaffrani W, et al. Successful treatment of clopidogrel hypersensitivity without drug interruption [abstract]. Circulation. 118,S891-892 (2008).

** This was the first study to describe a technique for managing clopidogrel hypersensitivity without drug interruption utilizing a short-course of corticosteroids and antihistamines.

${ }^{29}$ Vigo PG, MacDowell AL, Wedner HJ. Successful desensitization with clopidogrel after a positive skin test [abstract]. Ann Allergy Asthma Immunol. 94,132 (2005).

${ }^{30}$ ACC/AHA Task force on Practice Guidelines et al. 2007 Focused update of the ACC/AHA/SCAI 2005 guideline update for percutaneous coronary intervention. J Am Coll Cardiol. 51,172-209 (2008).

${ }^{31}$ Leon MB, Baim DS, Popma JJ, et al for the STARS Investigators. A clinical trial comparing three antithrombotic-drug regimens after coronary artery stenting. N Engl J Med. 339,1665-1671 (1998).

${ }^{32}$ Gent M, Easton JD, Hachinski VC, et al. The Canadian American ticlopidine study (CATS) in thromboembolic stroke. Lancet. 333,1215-1220 (1989). 
${ }^{33}$ Hass WK, Easton JD, Adams HP, et al. A randomized trial comparing ticlopidine hydrochloride with aspirin for the prevention of stroke in hig-risk patients. Ticlopidine aspirin stroke study group (TASS). $N$ Engl J Med. 321,501-507 (1989).

${ }^{34}$ Biondi-Zoccai GGL, Agostino P, Sangiorgi, GM, et al on behalf of the TRUE study investigators. Comparison of ticlopidine vs. clopidogrel in addition to aspirin after paclitaxel-eluting stent implantation: insights from the TRUE (Taxus in real-life usage evaluation) study. Int J Cardiol. 108,406-407 (2006). ${ }^{35}$ Ticlopidine hydrochloride tablet package insert. Toronto, Canada: Genpharm Inc, revised February 2002.

${ }^{36}$ Steinhubl SR, Tan WA, Foody JM, Topol EJ for the EPISTENT Investigators. Incidence and clinical course of thrombotic thrombocytopenic purpura due to diclopidine following coronary stenting. JAMA. 281,806-810 (1999).

${ }^{37}$ Wiviott SD, Braunwald E, McCabe CH, et al for the TRITON-TIMI 38 Investigators. Prasugrel versus clopidogrel in patients with acute coronary syndromes. N Engl J Med. 357,2001-2015 (2007).

* Including 13,608 patients, this trial demonstrates the efficacy of the newly available thienopyridine prasugrel in the treatment of patients with acute coronary syndrome and evaluates its safety profile. It does not investigate the utility of prasugrel as an alternative to clopidogrel in patients with clopidogrel hypersensitivity.

${ }^{38}$ Effient (prasugrel) package insert. Indianapolis, IN: Daiichi-Sankyo and Eli Lilly, revised July 2009.

${ }^{39}$ Walker NE, Fasano MB, Horwitz PA. Desensitization for the management of clopidogrel hypersensitivity: initial clinical experience. J Invasive Cardiol. 18,341-344 (2006).

${ }^{40}$ Camara MG, Almeda FQ. Clopidogrel (Plavix) desensitization: a case series. Catheter Cardiovasc Intervent. 65,525-527 (2005).

* This paper reports 3 patients experiencing clopidogrel hypersensitivity and describes a 7 hour protocol for desensitization.

${ }^{41}$ Makkar K, Wilensky RL, Julien MB, Herrmann HC, Spinler SA. Rash with both clopidogrel and ticlopidine in two patients following percutaneous coronary intervention with drug-eluting stents. Ann Pharmacother. 40,1204-1207 (2006). 
${ }^{42}$ Sharis PJ, Cannon CP, Loscalzo J. The antiplatelet effects of ticlopidine and clopidogrel. Ann Intern Med. 129,394-405 (1998).

${ }^{43}$ Bertrand ME, Legrand V, Boland J, Fleck et al. Randomized multicenter comparison of conventional anticoagulation versus antiplatelet therapy in unplanned and elective coronary stenting: the full anticoagulation versus aspirin and ticlopidine (FANTASTIC) study. Circulation. 98,1597-1603 (1998). ${ }^{44}$ Schomig A, Neumann FJ, Kastrati A, Schuhlen et al. A randomized comparison of antiplatelet and anticoagulant therapy after the placement of coronary-artery stents. N Engl J Med. 334,1084-1089 (1996).

${ }^{45} \mathrm{Ge}$ J, Han Y, Jiang H, et al. RACTS: a prospective randomized antiplatelet trial of cilostazol versus ticlopidine in patients undergoing coronary stenting: long-term clinical and angiographic outcome. $J$ Cardiovasc Pharmacol. 46,162-166 (2005).

${ }^{46}$ Lee SW, Park SW, Hong KM, et al. Comparison of cilostazol and clopidogrel after successful coronary stenting. Am J Cardiol. 95,859-862 (2005).

${ }^{47}$ Han Y, Wang S, Li Y, et al. Cilostazol improves long-term outcomes after coronary stent implantation Am Heart J. 150,e568.1-3 (2005).

${ }^{48}$ Durham SR. Allergen immunotherapy (desensitisation) for allergic diseases. Clin Med. 6,348-351 (2006).

** This article provides a good review of pathways involved in hypersensitivity reactions and the current understanding of immunologic mechanisms of desensitization.

${ }^{49}$ Lee-Wong M. Clopidogrel desensitzation [letter]. Ann Allery Asthma Immunol. 96,756-757 (2006).

${ }^{50}$ Walker NE, Fasano MB, Hobbs RA, Horwiz PA. Clopidogrel desensitization protocol for the treatment of thienopyridine hypersensitivity. Crit Pathways in Cardiol. 6,26-29 (2007).

* The authors detail a shorter 3 hour protocol used to successfully desensitize 13 patients with clopidogrel hypersensitivity.

${ }^{51}$ Owen P, Garner J, Hergott L, Page RL. Clopidogrel desensitization: case report and review of published protocols. Pharmacotherapy. 28,259-270 (2008).

** The authors detail a case report of a 58 year man who experienced clopidogrel hypersensitivity and his successfully desensitization as a means of reviewing published case reports of clopidogrel hypersensitivity and desensitization. 
${ }^{52}$ Kammer, RT. Successful clopidogrel desensitization after drug-eluting stent placement. J Invasive Cardiol. 21,134-135 (2009).

${ }^{53}$ Cheema AN, Mohammad A. Characterization of clopidogrel hypersensitivity reactions and response to oral steroids without clopidogrel discontinuation. J Am Coll Cardiol. 10(suppl A),A71 (2009). 